\title{
Erratum to: The upperbound of the volume expansion rate in a Lorentzian manifold
}

\author{
Jong Ryul Kim
}

Received: 8 August 2010 / Accepted: 14 September 2010 / Published online: 13 October 2010

(C) Springer Science+Business Media, LLC 2010

\section{Erratum to: Gen Relativ Gravit (2010) 42:403-412 DOI 10.1007/s10714-009-0860-4}

In the paper, we considered the following differential equation [1, pp. 7-8] along a geodesic $\gamma$ in a Riemannian manifold

$$
\theta^{\prime}+\theta^{2}+s^{M}-s\left(H_{t}\right)-\operatorname{Ric}\left(\gamma^{\prime}, \gamma^{\prime}\right)=0
$$

where $s\left(H_{t}\right)$ and $s^{M}$ denote, respectively, the scalar curvature of level hypersurface $H_{t}$ and $M$ at the point $\gamma(t)$ and $\theta(t)$ is the mean curvature of $H_{t}$ along $\gamma(t)$.

To calculate the upperbound of the volume expansion rate along a timelike geodesic $\gamma$ in a Lorentzian manifold, we used the above differential Eq. (1). But we neglected the sign when we take the trace of the Gauss equation (p. 408). Taking the sign of an unit speed timelike geodesic into account, Eq. (1) must be corrected to

$$
\theta^{\prime}+\theta^{2}+s^{M}-s\left(H_{t}\right)+3 \operatorname{Ric}\left(\gamma^{\prime}, \gamma^{\prime}\right)=0
$$

throughout the paper. As a consequence, we must correct the sentence "For the case of the negative Ricci curvature" (p. 405 below Eq. 7) to "For the case of the positive Ricci curvature".

I express my deep sorriness to the Scientific Community and Gen Relativ Gravit for such errors.

The online version of the original article can be found under doi:10.1007/s10714-009-0860-4.

J. R. Kim $(\varangle)$

Department of Mathematics, Kunsan National University, Kunsan 573-701, Republic of Korea e-mail:kimjr0@yahoo.com 


\section{Reference}

1. Eschenburg, J.-H., O’Sullivan, J.: Jacobi tensors and Ricci curvature. Math. Ann. 252, 1-26 (1980) 